\title{
ESTUDO SÔBRE A PESCA DE LAGOSTAS NO CEARÁ, DURANTE O ANO DE $1970^{(1)}$
}

\author{
Melquíades Pinto Paiva
}

\author{
Laboratório de Ciências do Mar \\ Universidade Federal do Ceará \\ Fortaleza - Ceará - Brasil
}

A exportação brasileira de caudas congeladas de lagostas, iniciada no ano de 1955 , alcançou o seu máximo anual em 1970 . No período 1955-1970, o total da exportação nacional do produto atingiu 20.122 toneladas métricas (tabela I) .

Durante o ano de 1970 , através do pôrto de Fortaleza, foram exportadas 2.036 toneladas métricas de caudas congeladas de lagostas, total superior ao de qualquer dos anos precedentes, e que representou $72,9 \%$ da exportação nacional do mesmo ano (tabela I) .

Na classificação das caudas congeladas de lagostas, exportadas pelo pôrto de Fortaleza durante o ano de 1970 (tabela II), merece destaque o fato da participação relativa do tipo 2-4 ter sido muito superior àquelas registradas para os oito anos precedentes, quando atingiu $48,1 \%$ das caixas exportadas (Paiva \& Moura, 1965 ; Paiva, 1966 , 1967 , 1968 , 1969 e 1970) .

No presente trabalho fazemos o estudo da pesca de lagostas no Estado do Ceará, durante o ano de 1970. As espécies Panulirus argus (Latreille) e Panulirus laevicauda (Latreille), as únicas que são comercialmente exploradas em tôda a região nordestina do Brasil, são consideradas em conjunto.

\section{DINÂMICA DA PESCA}

A produção de lagostas ao longo da costa cearense, durante o ano de 1970 , atingiu os totals de 16.335.611 indivíduos e 2.059.874 quilos de caudas frescas (tabela III), distribuída em ordem decrescente pelos trimestres, da maneira seguinte: segundo, primeiro, terceiro e quarto.

(1) - Trabalho realizado em decorrência de convênios firmados com a Superintendência do Desenvolvimento da Pesca (SUDEPE) e a Superintenpência do Desenvolvimento do Nordeste (SUDENE).
As áreas de pesca consideradas, já definidas anteriormente (Paiva, 1965b), são as que se seguem: Aracati - correspondendo aos municípios de Aracati, Beberibe e Cascavel; Fortaleza - correspondendo aos municípios de Aquiraz, Fortaleza e Caucaia; Paracuru -correspondendo aos municípios de São Gonçalo do Amarante, Paracuru e Trairi; Acaraú - correspondendo aos municípios de Itapipoca, Acaraú e Camocim (figura 1) .

Fizemos grandes amostragens dos desembarques de lagostas capturadas ao longo da costa cearense, com anotações sôbre os totais de caudas e quilos componentes de cada amostra, por trimestres e áreas de pesca (tabela IV) .

\section{T A B E I A I}

Exportação de caudas congeladas de lagostas, airavés do pôrto de Fortaleza (Estado do Ceará), comparada com a exportação nacional, durante os anos de 1955 a 1970 .

\begin{tabular}{|c|c|c|c|}
\hline \multirow{2}{*}{ Anos } & \multicolumn{2}{|c|}{ Toneladas métricas } & \multirow{2}{*}{$\begin{array}{l}\text { Participação } \\
\text { do Ceará }(\%)\end{array}$} \\
\hline & Ceará & BRASIL & \\
\hline $\begin{array}{l}1955 \\
1956 \\
1957 \\
1958 \\
1959 \\
1960 \\
1961 \\
1962 \\
1963 \\
1964 \\
1965 \\
1966 \\
1967 \\
1968 \\
1969 \\
1970\end{array}$ & $\begin{array}{r}40 \\
99 \\
189 \\
237 \\
390 \\
711 \\
1.265 \\
1.382 \\
1.102 \\
936 \\
771 \\
764 \\
870 \\
1.416 \\
1.916 \\
2.036\end{array}$ & $\begin{array}{r}40 \\
155 \\
346 \\
432 \\
616 \\
1.197 \\
1.740 \\
2.070 \\
1.778 \\
1 . .578 \\
1.181 \\
1.066 \\
974 \\
1.683 \\
2.473 \\
2.793\end{array}$ & $\begin{array}{r}100,0 \\
63,9 \\
54,6 \\
54,9 \\
63,3 \\
59,4 \\
72,7 \\
66,8 \\
62,0 \\
59,3 \\
65,3 \\
71,7 \\
89,3 \\
84,1 \\
77,5 \\
72,9\end{array}$ \\
\hline Total & 14.124 & 20.122 & 70,2 \\
\hline
\end{tabular}

Fon'e: Carteira do Comércio Exterior, Banco do Brasil S.A. 


\section{T A B E L A I I}

Exportação de caudas congeladas de lagostas, por tipos de exportação e em caixas de 10 libras, pelo pörto de Fortaleza, durante o ano de 1970 , com os correspondentes valôres relativos.

\begin{tabular}{c|r|r}
\hline $\begin{array}{c}\text { Tipos de exportação } \\
\text { (onças) }\end{array}$ & \multicolumn{2}{|c}{ Caixas exportadas } \\
\cline { 2 - 3 } & números & porcentagens \\
\cline { 2 - 3 } $2-4$ & 215.438 & 48,1 \\
$4-6$ & 131.277 & 29,3 \\
$6-8$ & 64.410 & 14,4 \\
$8-10$ & 27.860 & 6,2 \\
$10-12$ & 8.488 & 1,9 \\
$12-14$ & 488 & 0,1 \\
\hline Total & 447.961 & 100,0 \\
\hline
\end{tabular}

T A B E L A I I I

Dados sôbre a produção de lagostas ao longo da costa do Estado do Ceará, durante o ano de 1970 .

\begin{tabular}{|c|c|c|}
\hline Trimestres & Lagostas & Quilos * \\
\hline \multicolumn{3}{|c|}{ números absolutos } \\
\hline $\begin{array}{l}10^{\circ} \\
2 .^{\circ} \\
30^{\circ} \\
4 .^{\circ}\end{array}$ & $\begin{array}{l}4.175 .361 \\
5.993 .368 \\
3.392 .668 \\
2.774 .214\end{array}$ & $\begin{array}{l}524.143 \\
746.073 \\
421.487 \\
368.171\end{array}$ \\
\hline Ano & 16.335 .611 & 2.059 .874 \\
\hline \multicolumn{3}{|c|}{ números relativos } \\
\hline $\begin{array}{l}10^{\circ} \\
2 .^{\circ} \\
3.0 \\
4 .^{\circ}\end{array}$ & $\begin{array}{l}25,5 \\
36,7 \\
20,8 \\
17,0\end{array}$ & $\begin{array}{l}25,4 \\
36,2 \\
20,5 \\
17,9\end{array}$ \\
\hline Ano & 100,0 & 100,0 \\
\hline
\end{tabular}

* quilos de caudas frescas.

Obs.: através do pôrto de Fortaleza foram exportados 2.036.332 quilos de caudas congeladas de lagostas, durante o ano de 1970 .

Durante o ano de 1970 e com relaçäo à captura de lagostas, as áreas de pesca se distribuíram da seguinte maneira, em ordem de importância decrescente: primeiro trimestre - Paracuru, Aracati, Fortaleza e Acaraú; segundo trimestre - Aracati, Acaraú, Paracuru e Fortaleza; terceiro trimestre - Acaraú, Aracati, Paracuru e Fortaleza; quarto trimestre - Aracati, Acaraú, Paracuru e Fortaleza; ano - Aracati, Paracuru, Acaraú e Fortaleza.

Considerando cada área isoladamente, a pesca de lagostas mostrou importância decrescente entre os trimestres, a seguir discriminados: Aracati - segundo, primeiro, quarto e terceiro trimestres; Fortaleza - primeiro, segundo, quarto e terceiro trimestres; Paracuru - primeiro, segundo, terceiro e quarto trimestres; Acaraú - segundo, terceiro, quarto e primeiro trimestres.

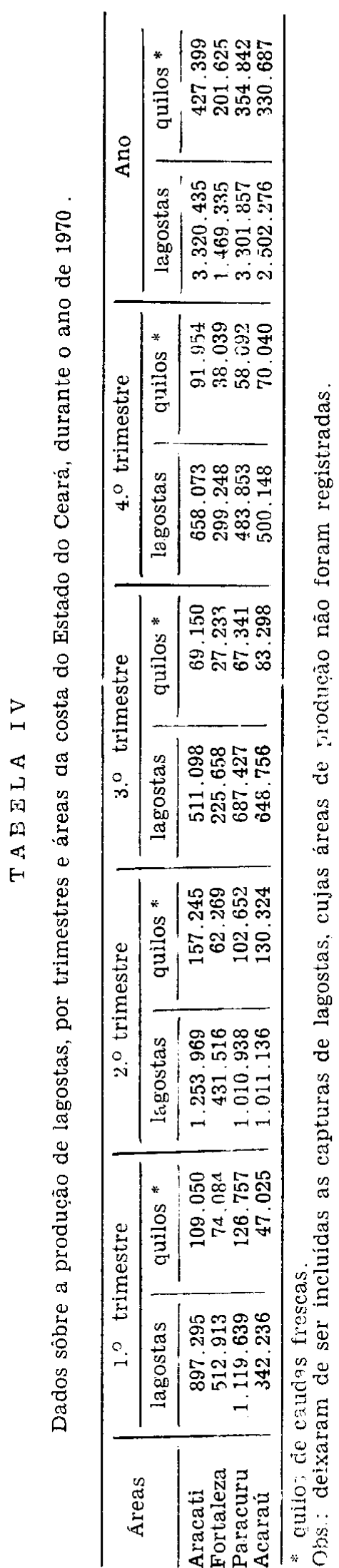

Com base nos dados das tabelas III e IV, calculamos os pesos médios de caudas de lagostas capturadas ao longo da costa cearense, referentes aos trimestres, ano e áreas de pesca (tabela V) . 


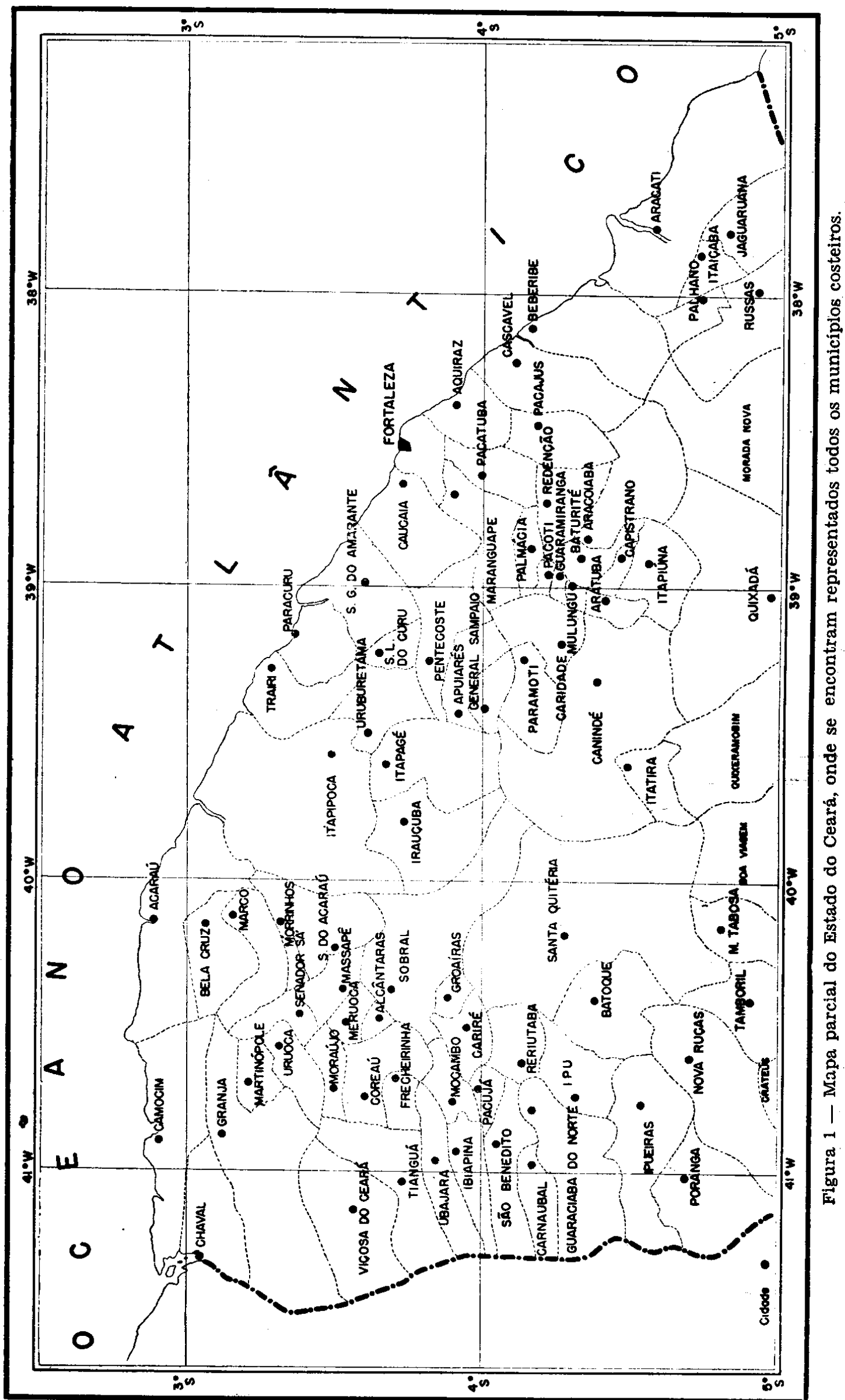


T A B E L A V

Pesos médios (g) das caudas de lagostas, capturadas ao longo da cost́a do Estado do Ceará, por trimestres e áreas de produção, bem como aquêles referentes às capturas totais, durante o ano de 1970

\begin{tabular}{|c|c|c|c|c|c|}
\hline \multirow{2}{*}{ Áreas } & \multicolumn{4}{|c|}{ Trimestres } & \multirow{2}{*}{ Ano } \\
\hline & 1.0 & $2 .^{\circ}$ & $3 .^{\circ}$ & $4 .^{\circ}$ & \\
\hline Aracati & 121 & 125 & 135 & 140 & 129 \\
\hline Fortaleza & 144 & 144 & 121 & $12 \pi$ & 137 \\
\hline Paracuru & 113 & 102 & 98 & 120 & 107 \\
\hline Acaraú & 137 & 129 & 128 & 140 & 132 \\
\hline Cearà & 126 & 124 & 124 & 133 & 126 \\
\hline
\end{tabular}

Durante o ano de 1970 , a média anual do pêso de caudas de lagostas, capturadas no Estado do Ceará, correspondeu a 126 gramas. Outras médias anuais conhecidas são as seguintes: em 1962 - 131 gramas, em 1963 146 gramas, em 1964 - 128 gramas, em 1965 - 131 gramas, em 1966 - 113 gramas, em 1967 - 127 gramas, em 1968 - 127 gramas, em 1969 - 133 gramas.

Considerando as áreas de pesca e os trimestres, em ordem decrescente do pêso médio de caudas de lagostas, temos as seguintes distribuições: áreas de pesca - Fortaleza, Acaraú, Aracati e Paracuru; trimestres - quarto, primeiro e segundo = terceiro. Com exceção para a área de Fortaleza, as maiores médias corresponderam ao quarto trimestre.

\section{DENSIDADE RELATIVA}

Os dados disponíveis sôbre a densidade relativa de lagostas resultam de anotações tomadas em diversos locais de desembarque, bem como de fôlhas de pesca da maioria dos barcos lagosteiros baseados no pôrto de Fortaleza, distribuídas e coletadas pela Superintencência do Desenvolvimento da Pesca.

A pesca de lagostas ao longo da costa cearense continuou, pràticamente, restrita à área limitada pela isobata de 50 metros, concentrando-se a partir de 20 metros de profundidade (figura 2).

Trabalhamos com blocos de 10 minutos de lado, que podem ser agrupados em blocos com 1 grau de lado. Usamos o sistema proposto pela Organização de Agricultura e Alimentação das Nações Unidas (FAO, 1968), para codificação de áreas marítimas, com algumas adaptações.

Tomemos para exemplo a posição $3^{\circ} 25^{\prime} \mathrm{S}$ - $38^{\circ} 17^{\prime} \mathrm{W}$, e vejamos a codificação para blocos de 10 minutos e 1 grau. $O$ bloco de $10 \mathrm{mi}$ nutos será codificado como 1.3.3/20.38/10, formado da seguinte maneira: bloco de 10 minutos de lado $=1$; quadrante sudoeste $=3$; latitude $3^{\circ} 25^{\prime} \mathrm{S}=3 / 20$; longitude $38^{\circ} 17^{\prime} \mathrm{W}=38 / 10$. O de 1 grau será codificado como 3.3.3.38, assim formado: bloco de 1 grau $=3$; quadrante sudoeste $=3$; latitude $3^{\circ} 25^{\prime} \mathrm{S}=3$; longitude $38^{\circ} 17^{\prime} \mathrm{W}=38$.

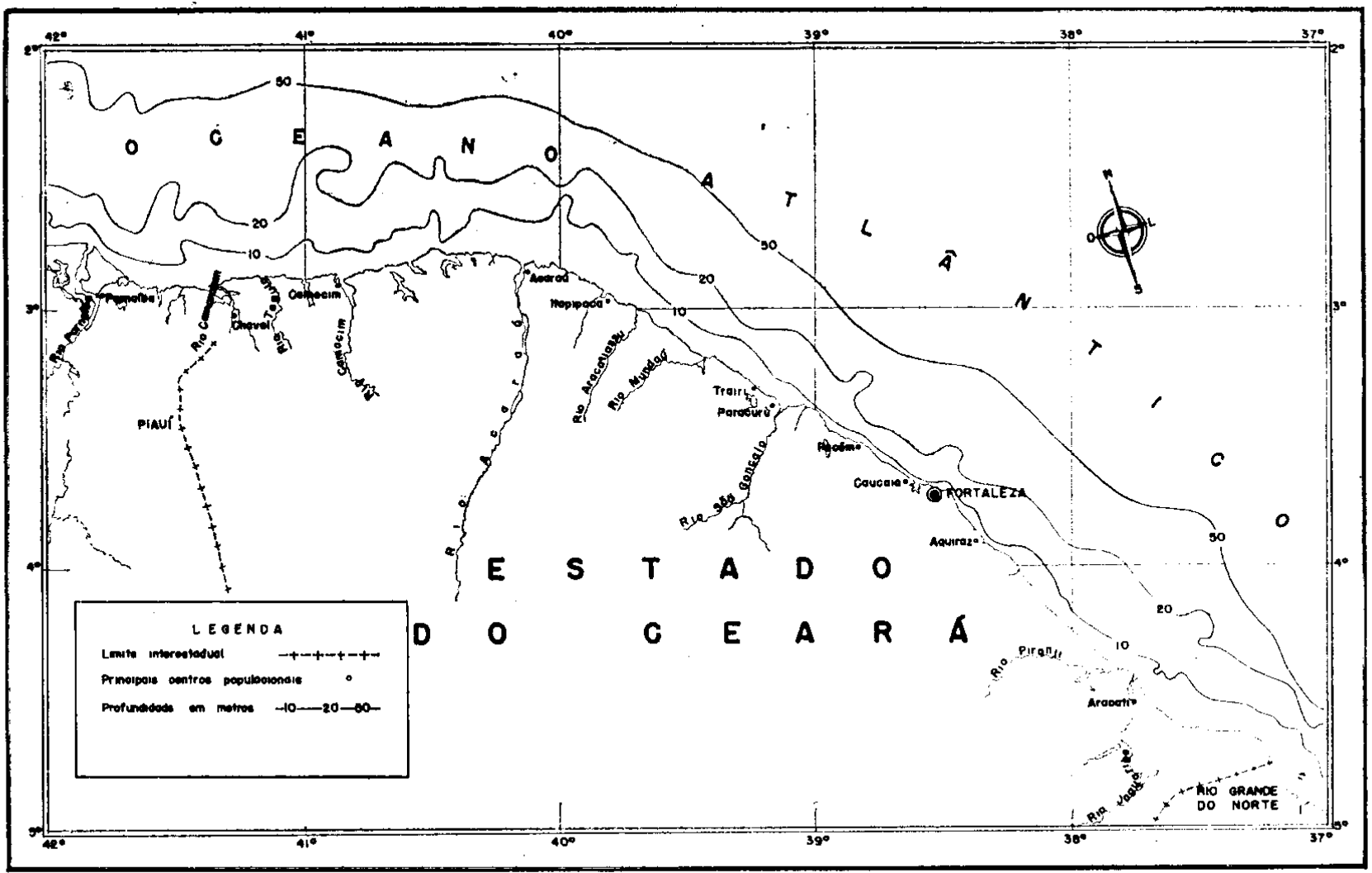

Figura 2 - Mapa da costa do Estado do Ceará, onde se encontram representadas as isobatas de 10,20 e 50 metros, da platalorma continental. 
Dados sôbre a densidade relativa de lagostas (individuos capturados por covo/dia) na costa cearense, durante o $10^{\circ}$ trimestre de 1970 .

\begin{tabular}{|c|c|c|c|}
\hline Blocos & $\begin{array}{l}\text { Covos } \\
\text { contro- } \\
\text { lados }\end{array}$ & $\begin{array}{l}\text { Lagostas } \\
\text { captura- } \\
\text { das }\end{array}$ & $\begin{array}{c}\begin{array}{c}\text { Densida- } \\
\text { des } \\
\text { relativas }\end{array} \\
\end{array}$ \\
\hline $\begin{array}{l}1.3 .3 / 50.37 / 20 \\
1.3 .3 / 50.37 / 40 \\
1.3 .3 / 40.37 / 50\end{array}$ & $\begin{array}{r}15.200 \\
4.180 \\
1.530\end{array}$ & $\begin{array}{r}17.237 \\
4.090 \\
1.200\end{array}$ & $\begin{array}{l}1,1 \\
1,0 \\
0,8\end{array}$ \\
\hline 3.3 .3 .37 & 20.910 & 22.527 & 1,1 \\
\hline $\begin{array}{l}1.3 .4 / 00.37 / 10 \\
1.3 .4 / 10.37 / 10 \\
1.3 .4 / 20.37 / 10 \\
1.3 .4 / 00.37 / 20 \\
1.3 .4 / 10.37 / 20 \\
1.3 .4 / 00.37 / 40\end{array}$ & $\begin{array}{r}900 \\
2.100 \\
2.000 \\
116.060 \\
7.040 \\
1.370\end{array}$ & $\begin{array}{r}505 \\
185 \\
1.600 \\
165.748 \\
5.215 \\
2.447\end{array}$ & $\begin{array}{l}0,6 \\
0,1 \\
0,8 \\
1,4 \\
0,7 \\
1,8\end{array}$ \\
\hline 3.3 .4 .37 & 129.470 & 175.700 & 1,4 \\
\hline 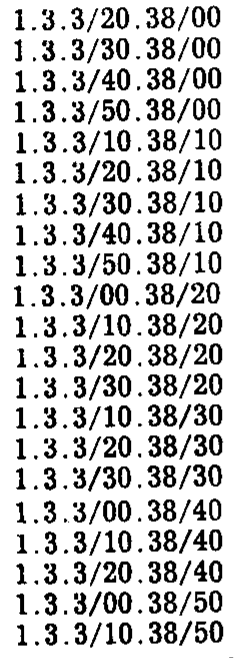 & $\begin{array}{r}2.040 \\
14.820 \\
16.670 \\
250 \\
55.050 \\
78.550 \\
87.022 \\
1.200 \\
756 \\
1.800 \\
39.570 \\
71.685 \\
980 \\
7.314 \\
12.610 \\
375 \\
28.200 \\
44.380 \\
15.580 \\
2.500 \\
1.120\end{array}$ & $\begin{array}{r}950 \\
14.300 \\
25.330 \\
126 \\
40.145 \\
90.283 \\
170.463 \\
2.700 \\
1.722 \\
2.230 \\
41.990 \\
128.207 \\
1.555 \\
6.835 \\
11.750 \\
1.340 \\
36.719 \\
54.040 \\
28.129 \\
7.250 \\
3.440\end{array}$ & $\begin{array}{l}0,5 \\
1,0 \\
1,5 \\
0,5 \\
0,7 \\
1,1 \\
2,0 \\
2,3 \\
2,3 \\
1,2 \\
1,1 \\
1,8 \\
1,6 \\
0,9 \\
0,9 \\
3,6 \\
1,3 \\
1,2 \\
1,8 \\
2,9 \\
3,1\end{array}$ \\
\hline 3.3 .3 .38 & 182.472 & 669.504 & 1,4 \\
\hline $1.3 .4 / 00.38 / 00$ & 720 & 1.590 & 2,2 \\
\hline 3.3 .4 .38 & 720 & 1.590 & 2,2 \\
\hline $\begin{array}{l}1.3 .2 / 40.39 / 00 \\
1.3 .2 / 50.39 / 00 \\
1.3 .2 / 30.39 / 10 \\
1.3 .2 / 40.39 / 10 \\
1.3 .2 / 50.39 / 10 \\
1.3 .2 / 20.39 / 20 \\
1.3 .2 / 30.39 / 20 \\
1.3 .2 / 40.39 / 20 \\
1.3 .2 / 50.39 / 20 \\
1.3 .2 / 20.39 / 30 \\
1.3 .2 / 30.39 / 30 \\
1.3 .2 / 50.39 / 30 \\
1.3 .2 / 30.39 / 40 \\
1.3 .2 / 40.39 / 40 \\
1.3 .2 / 10.39 / 50 \\
1.3 .2 / 20.39 / 50\end{array}$ & $\begin{array}{r}136.640 \\
5.215 \\
12.560 \\
66.030 \\
124.520 \\
49.310 \\
35.230 \\
5.050 \\
43.890 \\
4.550 \\
1.760 \\
2.560 \\
2.350 \\
300 \\
6.630 \\
72.900\end{array}$ & $\begin{array}{r}205.461 \\
10.025 \\
9.520 \\
79.649 \\
164.397 \\
58.321 \\
27.328 \\
6.100 \\
58.750 \\
5.310 \\
1.400 \\
7.355 \\
1.680 \\
500 \\
10.217 \\
93.050 \\
\end{array}$ & $\begin{array}{l}1,5 \\
1,9 \\
0,8 \\
1,2 \\
1,3 \\
1,2 \\
0,8 \\
1,2 \\
1,3 \\
1,2 \\
0,8 \\
2,9 \\
0,7 \\
1,7 \\
1,5 \\
1,3\end{array}$ \\
\hline 3.3.2.39 & 569.495 & 739.063 & 1,3 \\
\hline $\begin{array}{l}1.3 .3 / 00.39 / 00 \\
1.3 .3 / 00.39 / 10\end{array}$ & $\begin{array}{r}3.286 \\
56.700\end{array}$ & $\begin{array}{r}9.189 \\
132.205 \\
\end{array}$ & $\begin{array}{l}2,8 \\
2,3\end{array}$ \\
\hline 3.3.3.39 & 59.986 & 141.394 & 2,4 \\
\hline $\begin{array}{l}1.3 .2 / 10.40 / 00 \\
1.3 .2 / 20.40 / 00 \\
1.3 .2 / 30.40 / 00\end{array}$ & $\begin{array}{r}15.030 \\
2.275 \\
4.945\end{array}$ & $\begin{array}{r}10.414 \\
4.068 \\
14.342\end{array}$ & $\begin{array}{l}0,7 \\
1,8 \\
2,9\end{array}$ \\
\hline 3.3 .2 .40 & 22.250 & 28.824 & 1,3 \\
\hline Ceará & 285.303 & 778.602 & 1,4 \\
\hline
\end{tabular}

T A B E L A V I I

Dados sôbre a densidade relativa de lagostas (individuos capturados por covo/dia) na costa cearense, durante o $2 .^{\circ}$ trimestre de 1970 .

\begin{tabular}{|c|c|c|c|}
\hline Blocos & $\begin{array}{l}\text { Covus } \\
\text { contro- } \\
\text { lados }\end{array}$ & $\begin{array}{l}\text { Lagostas } \\
\text { captu- } \\
\text { radas }\end{array}$ & $\begin{array}{l}\text { Densida- } \\
\text { relativas }\end{array}$ \\
\hline $\begin{array}{l}1.3 .3 / 50.37 / 20 \\
1.3 .3 / 40.37 / 40 \\
1.3 .3 / 50.37 / 40\end{array}$ & $\begin{array}{r}6.78) \\
625 \\
3.120 \\
\end{array}$ & $\begin{array}{r}6.762 \\
519 \\
4.796 \\
\end{array}$ & $\begin{array}{l}1,0 \\
0,8 \\
1,5 \\
\end{array}$ \\
\hline 3.3 .3 .37 & 10.525 & 12.077 & 1,1 \\
\hline $\begin{array}{l}1.3 \cdot 4 / 20 \cdot 37 / 00 \\
1.3 .4 / 20 \cdot 37 / 10 \\
1.3 .4 / 00.37 / 20 \\
1.3 .4 / 10 \cdot 37 / 20 \\
1.3 .4 / 00.37 / 30 \\
1.3 .4 / 10.37 / 30 \\
1.3 .4 / 00.37 / 40\end{array}$ & $\begin{array}{r}8.764 \\
7.200 \\
33.370 \\
7.310 \\
397 \\
260 \\
1.170 \\
\end{array}$ & $\begin{array}{r}16.205 \\
15.500 \\
46.132 \\
5.570 \\
190 \\
180 \\
2.386\end{array}$ & $\begin{array}{l}1,8 \\
2,2 \\
1,4 \\
0,8 \\
0,5 \\
0,7 \\
2,0 \\
\end{array}$ \\
\hline 3.3 .4 .37 & 58.471 & 86.163 & 1,5 \\
\hline $1.3 .2 / 50.38 / 50$ & 1.870 & 1.200 & 0,6 \\
\hline 3.3 .2 .38 & 1.870 & 1.200 & 0,6 \\
\hline 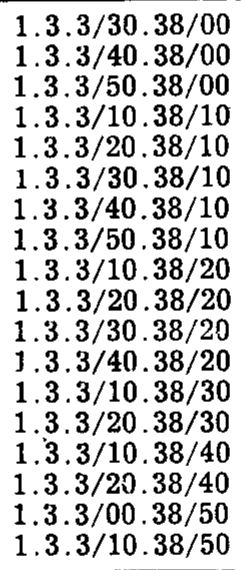 & $\begin{array}{r}17.42 J \\
6.420 \\
300 \\
16.130 \\
109.926 \\
130.349 \\
3.754 \\
756 \\
48.219 \\
13.180 \\
2.960 \\
320 \\
740 \\
2.560 \\
26.880 \\
2.730 \\
6.200 \\
8.750\end{array}$ & $\begin{array}{r}25.250 \\
12.500 \\
600 \\
20.995 \\
156.643 \\
290.350 \\
4.072 \\
1.722 \\
62.590 \\
86.843 \\
3.751 \\
414 \\
1.217 \\
4.900 \\
29.250 \\
2.971 \\
3.200 \\
19.700\end{array}$ & $\begin{array}{l}1,4 \\
1,9 \\
2,0 \\
1,3 \\
1,4 \\
2,2 \\
1,1 \\
2,3 \\
1,3 \\
1,8 \\
1,3 \\
1,3 \\
1,6 \\
1,9 \\
1,1 \\
1,1 \\
0,5 \\
2,3\end{array}$ \\
\hline 3.3 .3 .38 & 432.594 & 726.968 & 1,7 \\
\hline $\begin{array}{l}1.3 .2 / 40.39 / 00 \\
1.3 .2 / 50.39 / 00 \\
1.3 .2 / 30.39 / 10 \\
1.3 .2 / 40.39 / 10 \\
1.3 .2 / 50.39 / 10 \\
1.3 .2 / 20.39 / 20 \\
1.3 .2 / 30.39 / 20 \\
1.3 .2 / 40.39 / 20 \\
1.3 .2 / 50.39 / 20 \\
1.3 .2 / 20.39 / 30 \\
1.3 .2 / 30.39 / 30 \\
1.3 .2 / 40.39 / 30 \\
1.3 .2 / 50.39 / 30 \\
1.3 .2 / 20.39 / 40 \\
1.3 .2 / 30.39 / 40 \\
1.3 .2 / 40.39 / 40 \\
1.3 .2 / 10.39 / 50 \\
1.3 .2 / 20.39 / 50 \\
1.3 .2 / 30.39 / 50 \\
1.3 .2 / 40.39 / 50\end{array}$ & $\begin{array}{r}72.240 \\
27.580 \\
19.300 \\
23.450 \\
81.530 \\
3.700 \\
43.430 \\
1.950 \\
62.320 \\
22.710 \\
39.240 \\
14.950 \\
400 \\
14.450 \\
14.170 \\
2.400 \\
13.300 \\
120.755 \\
460 \\
1.000 \\
\end{array}$ & $\begin{array}{r}155.103 \\
63.375 \\
28.701 \\
30.741 \\
217.365 \\
8.207 \\
66.459 \\
2.800 \\
94.139 \\
25.590 \\
72.990 \\
60.373 \\
458 \\
17.290 \\
27.117 \\
9.456 \\
17.770 \\
228.812 \\
340 \\
1.703\end{array}$ & $\begin{array}{l}2,1 \\
2,3 \\
1,5 \\
1,3 \\
2,7 \\
2,2 \\
1,5 \\
1,4 \\
1,5 \\
1,1 \\
1,9 \\
4,0 \\
1,1 \\
1,2 \\
1,9 \\
3,9 \\
1,3 \\
1,9 \\
0,7 \\
1,7\end{array}$ \\
\hline 3.3 .2 .39 & 579.335 & $\overline{1.128 .789}$ & 1,9 \\
\hline $1.3 .3 / 00.39 / 00$ & 1.650 & 1.559 & 0,9 \\
\hline 3.3.3.39 & 1.650 & 1.559 & 0,9 \\
\hline
\end{tabular}




\begin{tabular}{c|r|r|r}
\hline $1.3 .2 / 10.40 / 00$ & 39.200 & 69.190 & 1,8 \\
$1.3 .2 / 20.40 / 00$ & 11.355 & 30.182 & 2,7 \\
$1.3 .2 / 30.40 / 00$ & 15.778 & 69.634 & 4,4 \\
$1.3 .2 / 10.40 / 10$ & 2.450 & 7.520 & 3,1 \\
$1.3 .2 / 20.40 / 10$ & 12.135 & 22.881 & 1,9 \\
$1.3 .2 / 30.40 / 10$ & 6.990 & 12.347 & 1,8 \\
$1.3 .2 / 10.40 / 20$ & 350 & 1.500 & 4,3 \\
$1.3 .2 / 10.40 / 40$ & 3.500 & 10.880 & 3,1 \\
$1.3 .2 / 20.40 / 40$ & 5.250 & 11.085 & 2,1 \\
$1.3 .2 / 30.40 / 40$ & 6.232 & 10.744 & 1,7 \\
$1.3 .2 / 00.40 / 50$ & 60.000 & 84.000 & 1,4 \\
$1.3 .2 / 10.40 / 50$ & 45.150 & 101.600 & 2,3 \\
$1.3 .2 / 20.40 / 50$ & 2.750 & 15.300 & 5,6 \\
$1.3 .2 / 40.40 / 50$ & 880 & 1.975 & 2,2 \\
\hline 3.3 .2 .40 & 212.020 & 448.838 & 2,1 \\
\hline $1.3 .2 / 10.41 / 10$ & 8.360 & 8.920 & 1,1 \\
$1.3 .2 / 30.41 / 10$ & 31.320 & 28.208 & 0,9 \\
$1.3 .2 / 10.41 / 20$ & 1.320 & 2.020 & 1,5 \\
$1.3 .2 / 20.41 / 20$ & 3.000 & 3.353 & 1,1 \\
$1.3 .2 / 30.41 / 20$ & 7.004 & 11.211 & 1,6 \\
$1.3 .2 / 40.41 / 20$ & 3.803 & 8.286 & 2,2 \\
\hline 3.3 .2 .41 & 54.807 & 61.998 & 1,1 \\
\hline Ceará & 1.351 .272 & 2.467 .592 & 1,8 \\
\hline
\end{tabular}

T A B E L A VIII

Dados sôbre a densidade relativa de lagostas (individios capturados por covo/dia) na costa cea rense, durante o $3 .^{\circ}$ trimestre de 1970 .

\begin{tabular}{|c|c|c|c|}
\hline Blocos & $\begin{array}{l}\text { Covos } \\
\text { contro } \\
\text { lados }\end{array}$ & $\begin{array}{l}\text { Lagostas } \\
\text { captu- } \\
\text { radas }\end{array}$ & $\begin{array}{c}\text { Densida- } \\
\text { des } \\
\text { relativas } \\
\end{array}$ \\
\hline $\begin{array}{l}1.3 .3 / 50.37 / 20 \\
1.3 .3 / 40.37 / 40 \\
1.3 .3 / 50.37 / 40 \\
\end{array}$ & $\begin{array}{l}2.600 \\
2.120 \\
7.360\end{array}$ & $\begin{array}{r}2.800 \\
2.155 \\
11.057 \\
\end{array}$ & $\begin{array}{l}1,1 \\
1,0 \\
1,5\end{array}$ \\
\hline 3.3 .3 .37 & 12.080 & 16.012 & 1,3 \\
\hline $\begin{array}{l}1.3 .4 / 30.37 / 00 \\
1.3 .4 / 20.37 / 10 \\
1.3 .4 / 30.37 / 10 \\
1.3 .4 / 00.37 / 20 \\
1.3 .4 / 10.37 / 20 \\
1.3 .4 / 20.37 / 20 \\
1.3 .4 / 00.37 / 30 \\
1.3 .4 / 10.37 / 30 \\
1.3 .4 / 20.37 / 30 \\
1.3 .4 / 00.37 / 40 \\
\end{array}$ & $\begin{array}{r}4.750 \\
9.310 \\
7.650 \\
16.880 \\
10.230 \\
2.800 \\
420 \\
4.825 \\
4.000 \\
2.960 \\
\end{array}$ & $\begin{array}{r}5.972 \\
5.945 \\
14.428 \\
15.330 \\
10.967 \\
3.917 \\
976 \\
5.373 \\
1.517 \\
2.467 \\
\end{array}$ & $\begin{array}{l}1,3 \\
0,6 \\
1,9 \\
0,9 \\
1,1 \\
1,4 \\
2,3 \\
1,1 \\
0,4 \\
0,8 \\
\end{array}$ \\
\hline 3.3 .4 .37 & 63.825 & 66.892 & 1,0 \\
\hline $\begin{array}{l}1.3 \cdot 3 / 20.38 / 00 \\
1.3 \cdot 3 / 30.38 / 00 \\
1.3 .3 / 40.38 / 00 \\
1.3 .3 / 10.38 / 10 \\
1.3 .3 / 20.38 / 10 \\
1.3 .3 / 30.38 / 10 \\
1.3 .3 / 10.38 / 20 \\
1.3 .3 / 20.38 / 20 \\
1.3 .3 / 30.38 / 20 \\
1.3 .3 / 10.38 / 30 \\
1.3 .3 / 20.38 / 30 \\
1.3 .3 / 10.38 / 40 \\
1.3 .3 / 20.38 / 40 \\
1.3 .3 / 30.38 / 40 \\
1.3 .3 / 10.38 / 50\end{array}$ & $\begin{array}{r}2.800 \\
5.720 \\
14.800 \\
781 \\
51.560 \\
73.910 \\
17.480 \\
33.965 \\
100 \\
720 \\
150 \\
54.816 \\
15.460 \\
200 \\
14.920\end{array}$ & $\begin{array}{r}1.500 \\
6.000 \\
20.660 \\
430 \\
47.656 \\
127.158 \\
15.750 \\
49.597 \\
145 \\
380 \\
170 \\
57.256 \\
25.248 \\
150 \\
27.450\end{array}$ & $\begin{array}{l}0,5 \\
1,0 \\
1,4 \\
0,6 \\
0,9 \\
1,7 \\
0,9 \\
1,5 \\
1,5 \\
0,5 \\
1,1 \\
1,0 \\
1,6 \\
0,8 \\
1,8\end{array}$ \\
\hline 3.3 .3 .38 & 287.382 & 379.550 & 1,3 \\
\hline
\end{tabular}

\begin{tabular}{|c|c|c|c|}
\hline $\begin{array}{l}1.3 .2 / 40.39 / 00 \\
1.3 .2 / 50.39 / 00 \\
1.3 .2 / 30.39 / 10 \\
1.3 .2 / 40.39 / 10 \\
1.3 .2 / 50.39 / 10 \\
1.3 .2 / 20.39 / 20 \\
1.3 .2 / 30.39 / 20 \\
1.3 .2 / 50.39 / 20 \\
1.3 .2 / 30.39 / 30 \\
1.3 .2 / 40.39 / 30 \\
1.3 .2 / 50.39 / 30 \\
1.3 .2 / 30.39 / 40 \\
1.3 .2 / 10.39 / 50 \\
1.3 .2 / 20.39 / 50 \\
1.3 .2 / 30.39 / 50 \\
1.3 .2 / 40.39 / 50 \\
\end{array}$ & $\begin{array}{r}155.805 \\
2.580 \\
1.100 \\
470 \\
4.410 \\
2.190 \\
44.300 \\
47.670 \\
22.490 \\
3.090 \\
1.400 \\
3.415 \\
300 \\
120.150 \\
8.340 \\
300 \\
\end{array}$ & $\begin{array}{r}207.763 \\
3.694 \\
682 \\
1.586 \\
5.355 \\
1.654 \\
80.708 \\
76.032 \\
24.648 \\
9.104 \\
2.134 \\
9.144 \\
130 \\
176.175 \\
8.377 \\
110\end{array}$ & $\begin{array}{l}1,3 \\
1,4 \\
0,6 \\
3,4 \\
1,2 \\
0,8 \\
1,8 \\
1,6 \\
1,1 \\
2,9 \\
1,5 \\
2,7 \\
0,4 \\
1,5 \\
1,0 \\
0,4\end{array}$ \\
\hline 3.3 .2 .39 & 418.010 & 607.296 & 1,5 \\
\hline $\begin{array}{l}1.3 .3 / 00.39 / 10 \\
1.3 .3 / 00.39 / 20 \\
\end{array}$ & $\begin{array}{l}800 \\
500\end{array}$ & $\begin{array}{r}1.125 \\
87 \\
\end{array}$ & $\begin{array}{l}1,4 \\
0,2\end{array}$ \\
\hline 3.3 .3 .39 & 1.300 & 1.212 & 0,9 \\
\hline $\begin{array}{l}1.3 .2 / 10.40 / 00 \\
1.3 .2 / 20.40 / 00 \\
1.3 .2 / 30.40 / 00 \\
1.3 .2 / 40.40 / 00 \\
1.3 .2 / 10.40 / 10 \\
1.3 .2 / 20.40 / 10 \\
1.3 .2 / 30.40 / 10 \\
1.3 .2 / 10.40 / 20 \\
1.3 .2 / 20.40 / 20 \\
1.3 .2 / 30.40 / 20 \\
1.3 .2 / 10.40 / 30 \\
1.3 .2 / 20.40 / 30 \\
1.3 .2 / 30.40 / 30 \\
1.3 .2 / 10.40 / 40 \\
1.3 .2 / 20.40 / 40 \\
1.3 .2 / 30.40 / 40 \\
1.3 .2 / 00.40 / 50 \\
1.3 .2 / 10.40 / 50 \\
1.3 .2 / 20.4050 \\
1.3 .2 / 30.40 / 50 \\
1.3 .2 / 40.40 / 50 \\
\end{array}$ & $\begin{array}{r}10.550 \\
17.550 \\
9.480 \\
300 \\
1.500 \\
900 \\
5.760 \\
300 \\
4.425 \\
3.500 \\
300 \\
3.300 \\
550 \\
300 \\
8.056 \\
1.750 \\
5.700 \\
35.880 \\
4.118 \\
780 \\
300\end{array}$ & $\begin{array}{r}14.601 \\
20.487 \\
14.725 \\
130 \\
1.536 \\
712 \\
11.897 \\
190 \\
7.527 \\
9.845 \\
110 \\
4.610 \\
485 \\
100 \\
8.000 \\
3.010 \\
3.864 \\
57.170 \\
3.053 \\
735 \\
135\end{array}$ & $\begin{array}{l}1,4 \\
1,2 \\
1,6 \\
0,4 \\
1,0 \\
0,8 \\
2,1 \\
0,6 \\
1,7 \\
2,8 \\
0,4 \\
1,4 \\
0,9 \\
0,3 \\
1,0 \\
1,7 \\
0,7 \\
1,6 \\
0,7 \\
0,9 \\
0,5\end{array}$ \\
\hline 3.3 .2 .40 & 115.299 & $\mathbf{1 6 2 . 9 2 2}$ & 1,4 \\
\hline $\begin{array}{l}1.3 .2 / 00.41 / 00 \\
1.3 .2 / 10.41 / 00 \\
1.3 .2 / 20.41 / 00 \\
1.3 .2 / 30.41 / 00 \\
1.3 .2 / 40.41 / 00 \\
1.3 .2 / 10.41 / 10 \\
1.3 .2 / 20.41 / 10 \\
1.3 .2 / 30.41 / 10 \\
1.3 .2 / 40.41 / 10 \\
1.3 .2 / 00.41 / 20 \\
1.3 .2 / 10.41 / 20 \\
1.3 .2 / 30.41 / 20 \\
\end{array}$ & $\begin{array}{r}2.400 \\
800 \\
270 \\
1.440 \\
400 \\
1.320 \\
4.200 \\
1.600 \\
120 \\
1.040 \\
4.500 \\
3.600 \\
\end{array}$ & $\begin{array}{r}3.640 \\
60 \\
215 \\
1.337 \\
25 \\
2.600 \\
7.460 \\
7.800 \\
140 \\
985 \\
6.065 \\
7.015 \\
\end{array}$ & $\begin{array}{l}1,5 \\
0,1 \\
0,8 \\
0,9 \\
0,1 \\
2,0 \\
1,8 \\
4,9 \\
1,2 \\
0,9 \\
1,3 \\
1,9 \\
\end{array}$ \\
\hline 3.3 .2 .41 & 21.690 & 37.342 & 1,7 \\
\hline Ceará & 919.586 & 1.271 .226 & 1,4 \\
\hline
\end{tabular}

T A B E L A I X

Dados sôbre a densidade relativa de lagostas (individuos capturados ror covo/dia) na costa cearense, duranie o $4 .^{\circ}$ trimestre de 1970 .

\begin{tabular}{c|r|r|r}
\hline Blocos & $\begin{array}{r}\text { Covos } \\
\text { contro- } \\
\text { lados }\end{array}$ & $\begin{array}{c}\text { Lagostas } \\
\text { captu- } \\
\text { radas }\end{array}$ & $\begin{array}{c}\text { Densida- } \\
\text { des } \\
\text { relativas }\end{array}$ \\
\cline { 1 - 2 } $1.3 .3 / 40.37 / 40$ & 4.730 & 4.065 & 0,9 \\
$1.3 .3 / 50.37 / 40$ & 700 & 527 & 0,8 \\
$1.3 .3 / 40.37 / 50$ & 260 & 285 & 1,1 \\
$1.3 .3 / 50.37 / 50$ & 720 & 670 & 0,9 \\
\hline 3.3 .3 .37 & 6.410 & 5.547 & 0,9 \\
\hline
\end{tabular}




\begin{tabular}{|c|c|c|c|}
\hline $\begin{array}{l}1.3 .4 / 40.37 / 00 \\
1.3 .4 / 20.37 / 10 \\
1.3 .4 / 30.37 / 10 \\
1.3 .4 / 10.37 / 20 \\
1.3 .4 / 20.37 / 20 \\
1.3 .4 / 00.37 / 30 \\
1.3 .4 / 20.37 / 30 \\
1.3 .4 / 00.37 / 40 \\
1.3 .4 / 10.37 / 40 \\
1.3 .4 / 20.37 / 40\end{array}$ & $\begin{array}{r}3.795 \\
2.220 \\
3.100 \\
6.720 \\
620 \\
6.080 \\
3.620 \\
2.965 \\
810 \\
1.400\end{array}$ & $\begin{array}{r}14.704 \\
1.511 \\
2.184 \\
5.885 \\
2.175 \\
5.111 \\
4.521 \\
753 \\
154 \\
3.668\end{array}$ & $\begin{array}{c}3,9 \\
0,7 \\
0,7 \\
0,9 \\
3,5 \\
0,8 \\
1,2 \\
0,3 \\
0,2 \\
2,6\end{array}$ \\
\hline 3.3 .4 .37 & 31.330 & 4.0 .666 & 1,3 \\
\hline 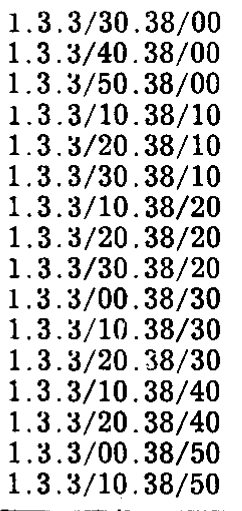 & $\begin{array}{r}1.860 \\
1.620 \\
2.665 \\
260 \\
1.020 \\
23.003 \\
4.070 \\
2.040 \\
6.000 \\
320 \\
2.240 \\
1.780 \\
1.800 \\
15.310 \\
1.600 \\
3.420\end{array}$ & $\begin{array}{r}575 \\
2.123 \\
1.829 \\
140 \\
1.036 \\
41.156 \\
3.680 \\
1.812 \\
3.477 \\
1.069 \\
820 \\
946 \\
1.526 \\
12.236 \\
2.067 \\
5.004\end{array}$ & $\begin{array}{l}0,3 \\
1,3 \\
0,7 \\
0,5 \\
1,0 \\
1,8 \\
0,9 \\
0,9 \\
0,6 \\
3,3 \\
0,4 \\
0,5 \\
0,8 \\
0,8 \\
1,3 \\
1,5\end{array}$ \\
\hline 3.3 .3 .38 & 69.008 & 79.496 & 1,2 \\
\hline $\begin{array}{l}1.3 .2 / 40.39 / 00 \\
1.3 .2 / 50.39 / 00 \\
1.3 .2 / 40.39 / 10 \\
1.3 .2 / 50.39 / 10 \\
1.3 .2 / 20.39 / 20 \\
1.3 .2 / 30.39 / 20 \\
1.3 .2 / 40.39 / 20 \\
1.3 .2 / 50.39 / 20 \\
1.3 .2 / 30.39 / 30 \\
1.3 .2 / 40.39 / 30 \\
1.3 .2 / 50.39 / 30 \\
1.3 .2 / 20.39 / 40 \\
1.3 .2 / 30.39 / 40 \\
1.3 .2 / 40.39 / 40 \\
1.3 .2 / 10.39 / 50 \\
1.3 .2 / 20.39 / 50 \\
1.3 .2 / 30.39 / 50 \\
1.3 .2 / 40.39 / 50\end{array}$ & $\begin{array}{r}124.249 \\
2.120 \\
3.800 \\
2.600 \\
440 \\
1.000 \\
5.390 \\
3.000 \\
7.420 \\
2.610 \\
2.040 \\
2.320 \\
3.670 \\
2.110 \\
8.100 \\
12.880 \\
4.928 \\
120\end{array}$ & $\begin{array}{r}169.146 \\
1.193 \\
3.410 \\
2.773 \\
574 \\
402 \\
5.625 \\
2.590 \\
8.645 \\
3.376 \\
3.745 \\
1.456 \\
8.108 \\
7.580 \\
10.939 \\
8.487 \\
8.629 \\
109\end{array}$ & $\begin{array}{l}1,4 \\
0,6 \\
0,9 \\
1,1 \\
1,3 \\
0,4 \\
1,0 \\
0,9 \\
1,2 \\
1,3 \\
1,8 \\
0,6 \\
2,2 \\
3,6 \\
1,4 \\
0,7 \\
1,8 \\
0,9\end{array}$ \\
\hline 3.3.2.39 & 188.797 & 246.787 & 1,3 \\
\hline $\begin{array}{l}1.3 .3 / 00.39 / 00 \\
1.3 .3 / 00.39 / 10\end{array}$ & $\begin{array}{l}1.370 \\
3.960\end{array}$ & $\begin{array}{l}1.345 \\
5.073\end{array}$ & $\begin{array}{l}1,0 \\
1,3\end{array}$ \\
\hline 3.3.3.39 & 5.330 & 6.418 & 1,2 \\
\hline $\begin{array}{l}1.3 \cdot 2 / 10.40 / 00 \\
1.3 .2 / 20.40 / 00 \\
1.3 .2 / 30.40 / 00 \\
1.3 .2 / 20.40 / 10 \\
1.3 .2 / 30.40 / 10 \\
1.3 .2 / 10.40 / 20 \\
1.3 .2 / 20.40 / 20 \\
1.3 .2 / 30.40 / 20 \\
1.3 .2 / 10.40 / 30 \\
1.3 .2 / 20.40 / 30 \\
1.3 .2 / 30.40 / 30 \\
1.3 .2 / 10.40 / 40 \\
1.3 .2 / 20.40 / 40 \\
1.3 .2 / 30.40 / 40 \\
1.3 .2 / 10.40 / 50 \\
1.3 .2 / 20.40 / 50 \\
1.3 .2 / 30.40 / 50\end{array}$ & $\begin{array}{r}9.590 \\
6.984 \\
17.800 \\
7.100 \\
700 \\
8.120 \\
5.000 \\
1.680 \\
815 \\
11.630 \\
6.280 \\
6.500 \\
10.980 \\
2.790 \\
10.710 \\
8.510 \\
12.160\end{array}$ & $\begin{array}{r}11.955 \\
8.984 \\
38.792 \\
14.410 \\
400 \\
16.354 \\
8.224 \\
2.090 \\
1.337 \\
24.230 \\
9.814 \\
12.377 \\
12.285 \\
2.740 \\
15.886 \\
7.666 \\
19.334\end{array}$ & $\begin{array}{l}1,2 \\
1,3 \\
2,2 \\
2,0 \\
0,6 \\
2,0 \\
1,6 \\
1,2 \\
1,6 \\
2,1 \\
1,6 \\
1,9 \\
1,1 \\
1,0 \\
1,5 \\
0,9 \\
1,6\end{array}$ \\
\hline 3.3 .2 .40 & 127.349 & 206.878 & 1,6 \\
\hline
\end{tabular}

Nas tabelas VI a IX apresentamos os índices de densidade relativa, correspondentes aos blocos onde conseguimos controlar captu-

\begin{tabular}{c|r|r|r}
\hline $1.3 .2 / 10.41 / 00$ & 2.280 & 4.615 & 2,0 \\
$1.3 .2 / 20.41 / 00$ & 2.000 & 4.600 & 2,3 \\
$1.3 .2 / 30.41 / 00$ & 9.110 & 19.810 & 2,2 \\
$1.3 .2 / 10.41 / 10$ & 2.200 & 1.288 & 0,6 \\
$1.3 .2 / 20.41 / 10$ & 830 & 1.000 & 1,2 \\
$1.3 .2 / 30.41 / 10$ & 3.750 & 5.760 & 1,5 \\
$1.3 .2 / 40.41 / 10$ & 1.500 & 2.855 & 1,9 \\
$1.3 .2 / 20.41 / 20$ & 3.500 & 3.184 & 0,9 \\
$1.3 .2 / 30.41 / 20$ & 900 & 1.180 & 1,3 \\
$1.3 .2 / 40.41 / 20$ & 1.060 & 1.158 & 1,1 \\
\hline 3.3 .2 .41 & 27.140 & 45.450 & 1,7 \\
\hline Ceará & 455.364 & 631.242 & 1,4 \\
\hline
\end{tabular}

T A B E L A $\mathrm{X}$

Dados sôbre a densidade relativa de lagostas (individuos capturados por covo/dia) na costa cearense, por faixas de longitudes, trimestres e ano de 1970 .

\begin{tabular}{|c|c|c|c|}
\hline $\begin{array}{l}\text { Faixas de } \\
\text { longitudes }\end{array}$ & $\left|\begin{array}{c}\text { Covos con }- \\
\text { trolados }\end{array}\right|$ & $\begin{array}{c}\text { Lagostas } \\
\text { capturadas }\end{array}$ & $\begin{array}{c}\text { Densidades } \\
\text { relativaf }\end{array}$ \\
\hline \multicolumn{4}{|c|}{$10^{\circ}$ trimestre } \\
\hline $\begin{array}{l}37^{\circ} \mathrm{W}-38^{\circ} \mathrm{W} \\
38^{\circ} \mathrm{W}-39^{\circ} \mathrm{W} \\
39^{\circ} \mathrm{W}-40^{\circ} \mathrm{W} \\
40^{\circ} \mathrm{W}-41^{\circ} \mathrm{W}\end{array}$ & $\begin{array}{r}150.380 \\
483.192 \\
629.481 \\
22.250\end{array}$ & $\begin{array}{r}198.227 \\
671.094 \\
880.457 \\
28.824\end{array}$ & $\begin{array}{l}1,3 \\
1,4 \\
1,4 \\
1,3\end{array}$ \\
\hline \multicolumn{4}{|c|}{ 2. ${ }^{\circ}$ trimestre } \\
\hline $\begin{array}{l}37^{\circ} \mathrm{W}-38^{\circ} \mathrm{W} \\
38^{\circ} \mathrm{W}-39^{\circ} \mathrm{W} \\
39^{\circ} \mathrm{W}-40^{\circ} \mathrm{W} \\
40^{\circ} \mathrm{W}-41^{\circ} \mathrm{W} \\
41^{\circ} \mathrm{W}-42^{\circ} \mathrm{W}\end{array}$ & $\begin{array}{r}68.996 \\
134.464 \\
580.985 \\
212.020 \\
54.807\end{array}$ & $\begin{array}{r}98.240 \\
728.168 \\
1.130 .348 \\
448.838 \\
61.998\end{array}$ & $\begin{array}{l}1,4 \\
1,7 \\
1,9 \\
2,1 \\
1,1\end{array}$ \\
\hline \multicolumn{4}{|c|}{ 3..$^{\circ}$ trimestre } \\
\hline $\begin{array}{l}37^{\circ} \mathrm{W}-38^{\circ} \mathrm{W} \\
38^{\circ} \mathrm{W}-39^{\circ} \mathrm{W} \\
39^{\circ} \mathrm{W}-40^{\circ} \mathrm{W} \\
40^{\circ} \mathrm{W}-41^{\circ} \mathrm{W} \\
41^{\circ} \mathrm{W}-42^{\circ} \mathrm{W}\end{array}$ & $\begin{array}{r}75.905 \\
287.382 \\
419.310 \\
115.299 \\
21.690\end{array}$ & $\begin{array}{r}82.904 \\
379.550 \\
608.508 \\
162.922 \\
37.342\end{array}$ & $\begin{array}{l}1,1 \\
1,3 \\
1,5 \\
1,4 \\
1,7\end{array}$ \\
\hline \multicolumn{4}{|c|}{$4 .^{\circ}$ trimestre } \\
\hline $\begin{array}{l}37^{\circ} \mathrm{W}-38^{\circ} \mathrm{W} \\
38^{\circ} \mathrm{W}-39^{\circ} \mathrm{W} \\
39^{\circ} \mathrm{W}-40^{\circ} \mathrm{W} \\
40^{\circ} \mathrm{W}-41^{\circ} \mathrm{W} \\
41^{\circ} \mathrm{W}-42^{\circ} \mathrm{W}\end{array}$ & $\begin{array}{r}37.740 \\
69.008 \\
194.127 \\
127.349 \\
2.140\end{array}$ & $\begin{array}{r}46.213 \\
79.496 \\
253.205 \\
206.878 \\
45.450\end{array}$ & $\begin{array}{l}1,2 \\
1,2 \\
1,3 \\
1,6 \\
1,7\end{array}$ \\
\hline \multicolumn{4}{|c|}{ Ano } \\
\hline $\begin{array}{l}37^{\circ} \mathrm{W}-38^{\circ} \mathrm{W} \\
38^{\circ} \mathrm{W}-39^{\circ} \mathrm{W} \\
39^{\circ} \mathrm{W}-40^{\circ} \mathrm{W} \\
40^{\circ} \mathrm{W}-41^{\circ} \mathrm{W} \\
41^{\circ} \mathrm{W}-42^{\circ} \mathrm{W}\end{array}$ & $\begin{array}{r}333.021 \\
1.274 .046 \\
1.823 .903 \\
176.918 \\
103.637\end{array}$ & $\begin{array}{r}425.584 \\
1.858 .308 \\
2.872 .518 \\
847.462 \\
144.790\end{array}$ & $\begin{array}{l}1,3 \\
1,5 \\
1,6 \\
1,8 \\
1,4\end{array}$ \\
\hline Ceará & 4.011 .525 & 6.148 .662 & 1,5 \\
\hline
\end{tabular}

ras de lagostas ao longo da costa cearense, durante os trimestres do ano de 1970 . Como decorrência da extensão da costa, calculamos os índices de densidade relativa por faixas de longitudes, nos trimestres e no ano considerado (tabela $\mathrm{X}$ ).

$\mathrm{Na}$ faixa entre as longitudes $37^{\circ} \mathrm{W}-$ $38^{\circ} \mathrm{W}$, os valôres encontrados para as capturas expressas em covo/dia foram 1,3 , 1,4 , 1,1 e 1,2 lagostas, respectivamente para o primeiro, segundo, terceiro e quarto trimestres. $O$ índice anual de densidade relativa correspondeu a 1,3 lagostas. 


\section{T A B E L A X I}

Estimativas do esfôrço de pesca (covos/dia) empregado nas pescarias de lagostas ao longo da costa do Estado do Ceará, por trimestres e ano de 1970

\begin{tabular}{c|c}
\hline Trimestres & Covos/dia \\
\hline $10^{\circ}$ & 2.982 .400 \\
$2 .^{\circ}$ & 3.329 .648 \\
$3 .^{\circ}$ & 2.423 .334 \\
$4 .^{\circ}$ & 1.981 .581 \\
\hline Ano & 10.716 .963
\end{tabular}

Obs.: por cálculo direto obtemos o valor anual do esfôrço empregado, correspondendo a 10.890.407 covos/dia.

$\mathrm{Na}$ faixa entre as longitudes $38^{\circ} \mathrm{W}-$ $39^{\circ} \mathrm{W}$, os valôres encontrados para as capturas expressas em covo/dia foram $1,4,1,7,1,3$ e 1,2 lagostas, respectivamente para o primeiro, segundo, terceiro e quarto trimestres. $\mathrm{O}$ índice anual de densidade relativa correspondeu a 1,5 lagostas.

$\mathrm{Na}$ faixa entre as longitudes $39^{\circ} \mathrm{W}$ $40^{\circ} \mathrm{W}$, os valôres encontrados para as capturas expressas em covo/dia foram $1,4,1,9,1,5$ e 1,3 lagostas, respectivamente para o primeiro, segundo, terceiro e quarto trimestres. $O$ írdice anual de densidade relativa correspondeu a 1,6 lagostas.

$\mathrm{Na}$ faixa entre as longitudes $40^{\circ} \mathrm{W}-$ $411^{\circ} \mathrm{W}$, os valôres encontrados para as capturas expressas em covo/dia foram 1,3 , 2,1 , 1,4 e 1,6 lagostas, respectivamente para o primeiro, segundo, terceiro e quarto trimestres. $O$ índice anual de densidade relativa corresprindeu a 1,8 lagostas.

$\mathrm{Na}$ faixa entre as longitudes $41^{\circ} \mathrm{W}$ $42^{\circ} \mathrm{W}$, os valôres encontrados para as capturas expressas em covo/dia foram 1,1 e 1,7 lagostas, respectivamente para o segundo, terceiro e quarto trimestres. $O$ índice anual de densidade relativa correspondeu a 1,4 lagostas.

No primeiro trimestre, as melhores pescarias se realizaram entre as longitudes $38^{\circ} \mathrm{W}-$ $40^{\circ} \mathrm{W}$; no segundo trimestre, entre as longitudes $40^{\circ} \mathrm{W}-41^{\circ} \mathrm{W}$; no terceiro e quarto trimestres, entre as longitudes $41^{\circ} \mathrm{W}-42^{\circ} \mathrm{W}$. Em têrmos anuais, tais pescarias se efetuaram entre as longitudes $40^{\circ} \mathrm{W}-41^{\circ} \mathrm{W}$.

$O$ índice anual de densidade relativa, expresso em lagostas capturadas por covo/dia, referente ao Estado do Ceará, correspondeu a 1,5 lagostas em 1970. Outros índices anuais conhecidos são os seguintes: em $1964-8,4$ lagostas, em 1965 - 3,2 lagostas, em $1966-$ 2,5 lagostas, em 1967 - 1,9 lagostas, em 1968 - 2,0 lagostas, em $1969-1,3$ lagostas (Paiva, $1965 a, 1966,1967,1968,1969$ e 1970).

\section{ESFÔRÇO DE PESCA}

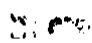

O esfôrço empregado na pesca de lagos- tas no Estado do Ceará, no ano de 1970 , correspondeu a cêrca de 10.800 .000 covos/dia (tabela XI). As estimativas feitas para os três anos precedentes foram as seguintes: em 1967 -4.000 .000 covos/dia, em $1968-6.500 .000$ covos/dia, em $1969-11.500 .000$ covos/dia (Paiva, 1968 , 1969 e 1970) .

Em ordem decrescente de importância, a ciistribuição do esfôrço de pesca, aplicado na exploração lagosteira ao longo da costa cearense, durante os trimestres de 1970 , foi a seguinte: segundo, primeiro, terceiro e quarto.

\section{CONSIDERAÇÕES FINAIS}

Da análise global dos dados, temos a destacar alguns aspectos que merecem especial atenção.

A produção de lagostas ao longo da costa cearense alcançou em 1970 o seu máximo anual, com elevada captura de indivíduos menores, o que se reflete na alta participação do tipo 2-4 nas exportações e na baixa média anual do pêso médio das caudas frescas.

Em virtude do aumento da produtividade, expressa em lagostas capturadas por covo/ dia, podemos explicar a pequena redução verificada no esfôrço de pesca empregado, com reilação ao ano anterior, ao lado do aumento observado no volume das capturas.

\section{S U M M A R Y}

In this paper we study the spiny lobster fishery in the State of Ceará (Brazil) during 1970 . The species Panulirus argus (Latreille) and Panulirus laevicauda (Latreille), the only ones that comprise the commercial landings in the whole northeastern region of Brazil, are together considered.

A total production of $2,059,874$ kilos of fresh tails was reached, corresponding to 16,335,611 spiny lobsters captured. Through the port of Fortaleza 2,036,332 kilos of spiny lobster frozen tails were exported.

The mean weight of the spiny lobster tails for the State of Ceará during 1970 corresponcied to 126 grams. The annual mean of relative density was 1.5 spiny lobsters caught per trap/day. The fishing effort reached about $10,800,000$ traps/day.

The spiny lobsters production in the State of Ceará during 1970 was higher than in the previous years, since 1955 when the exploitation started. A large number of small individuals was captured, which resulted in the high participation of 2-4 ounces type in the exportation and low annual mean of the fresh tails weight.

The productivity increase per trap/day explains the small reduction in the fishing 
effort besides the larger capture volume, in relation to the previous year.

\section{REFERENCIAS BIBLIOGRÁFICAS}

FAO - 1968 - Un sistema de codificación de area maritima. CARPAS W.G./3 D. Trab., Rio de Janeiro, (2) : 1-3.

Paiva, M. P. - $1965 a$ - Dados sôbre a densidade relativa de lagostas na costa cearense em 1964. Arq. Est. Biol. Mar. Univ. Ceará, Fortaleza, 5 (1) : 1-9, 3 figs.

Paiva, M. P. - $1965 b$ - Dinâmica da pesca de lagostas no Ceará. Arq. Est. Biol. Mar. Univ. Ceará, Fortaleza, 5 (2): 151-174, 5 figs.

Paiva, M. P. - 1966 - Estudo sôbre a pesca de lagostas no Ceará, durante o ano de 1965. Arq. Est.
Biol. Mar. Univ. Fed, Ceará, Fortaleza, 6 (2) : 147-165, 9 figs

Paiva, M. P. - 1967 - Estudo sôbre a pesca de lagostas no Ceará, durante o ano de 1966. Arq Est. Biol. Mar. Univ. Fed. Ceará, Fortaleza, 7 (2) : 123-142, 9 figs.

Paiva, M. P. - 1968 - Estudo sôbre a pesca de lagostas no Ceará, durante o ano de 1967. Arq. Est. Biol. Mar. Univ. Fed. Ceará, Fortaleza, 8 (1): 47-64, 5 figs.

Paiva, M. P. - 1969 - Estudo sôbre a pesca de lagostas no Ceará, durante o ano de 1968. Arq. Ciên. Mar, Fortaleza, 9 (1) : 41-55, 5 figs.

Paiva, M. P. - 1970 - Estudo sôbre a pesca de lagostas no Ceará, durante o ano de 1969 . Arq. Ciên. Mar, Fortaleza, 10 (1): 91-103, 5 figs.

Paiva, M. P. \& Moura, S. J. C. - 1965 - Sôbre a classificação da exportação nacional de caudas de lagostas. Bol. Est. Biol. Mar. Univ. Cul "í, Hortaleza, (9) : 1-8, 1 fig. 